Revista Científica de FAREM-Estelí

Medio ambiente, tecnologia y desarrollo humano

Año 10 | Edición especial: artículos de revisión documental

ISSN: 2305-5790

https://rcientificaesteli.unan.edu.ni

DOI: https://doi.org/10.5377/farem.v0i0.11606

\section{Breve revisión conceptual sobre la evaluación de los resultados académicos en el sistema educativo}

\section{Brief conceptual review on the evaluation of academic results in the educational system}

\section{Andrea Lucía Córdoba Peralta}

Estudiante de doctorado en Gestión y Calidad de la Investigación Científica.

II Edición. Universidad Nacional Autónoma de Nicaragua, UNAN-Managua,

Facultad Regional Multidisciplinaria de Estelí (FAREM-Estelí)

https://orcid.org/0000-0002-4829-5990

aluciacp06@yahoo.com

\section{Emilio Martín Lanuza Saavedra}

Docente Titular (TD). UNAN-Managua/FAREM-Estelí. Doctor en Gestión y Calidad de la Educación

https://orcid.org/0000-0002-0971-0100

emilio.lanuza@gmail.com

\section{RESUMEN}

En el presente artículo de revisión documental, se realizó un estudio teórico de la evaluación en la educación. Las principales fuentes consultadas fueron libros y artículos de revistas. En la evaluación existen diversas definiciones, funciones y prácticas que han generado debates a lo largo de la historia y aún siguen vigentes en los sistemas educativos. En este artículo, se incluyen algunos conceptos de evaluación, características, modalidades y paradigmas. Se plantea la consideración de la elaboración del informe de rendimiento académico como una herramienta de realimentación para el análisis de los aspectos de la actividad educativa que resultaron satisfactorios y es necesario fortalecer y aquellos que serán precisos modificar. Es un intento de ampliar la mirada de la evaluación en el aula, a partir del rendimiento académico, que permita transformar las prácticas evaluativas que implementan los docentes. Para dar continuidad a dichos resultados, no se limita únicamente al proceso de medición con fines de acreditación del semestre académico o promoción del año lectivo. Esto implica, establecer estrategias y acciones de mejora continua en el proceso educativo en beneficio del aprendizaje de los estudiantes, justo a partir del análisis crítico de los resultados académicos.
RECIBIDO

$26 / 03 / 2021$

ACEPTADO

19/052021

\section{PALABRAS CLAVE}

Evaluación; aprendizajes; rendimiento académico. 


\section{ABSTRACT}

In this documentary review article, a theoretical study of evaluation in education was carried out. The main sources consulted were books, journal articles. There are several definitions, functions and practices in evaluation that have generated debates throughout history and are still in force in educational system. In this article, some assessment concepts, characteristics, modalities and paradigms are included. It considers the elaboration of the academic performance report as a feedback tool for the analysis of the aspects of the educational activity that were satisfactory and need to be strengthened and those that need to be modified. It is an attempt to expand the view of the classroom evaluation, based on academic performance, which allow transforming the evaluative practice implemented by teachers. In order to give continuity to these results, it is not limited only to the measurement process for the purpose of accreditation of the academic semester or promotion of academic year. This implies, stablishing strategies and actions for continuous improvement in the educational process for the benefit of students learning, based on the critical analysis of the academic results.

\section{KEYWORDS}

Evaluation; learning; academic performance. 


\section{INTRODUCCIÓN}

La educación es un factor en la transformación y el desarrollo del ser humano, influye en el avance y progreso de las personas y por ende de la sociedad. El sistema educativo lleva consigo la etapa de la evaluación, a través de la cual se observa, recoge y se analizan datos significativos, para la toma de decisiones pertinentes y oportunas en la mejora del aprendizaje de los estudiantes. La evaluación es un proceso que compromete a todos los agentes del sistema educativo.

Existen diferentes concepciones para la evaluación. En el ámbito educativo, se define como: "un proceso sistemático de recogida de información, con el $\square$ n de determinar el mérito y el valor de un objeto y permitir la toma de decisiones para la mejora" (De Miguel, 2012, p.16). Se enfatiza a la evaluación como un proceso que se lleva a cabo para la toma de decisiones que aporte a la mejora educativa.

Uno de los componentes que forman parte de la evaluación de los resultados del proceso educativo es el rendimiento académico de los estudiantes. En un informe evaluativo de rendimiento académico se reflejan las valoraciones de docentes, estudiantes y los directivos de la institución educativa a nivel cuantitativo y cualitativo.

El propósito del presente estudio consiste en reflexionar teóricamente sobre la evaluación de los resultados académicos que se realiza al finalizar el semestre y/o año lectivo. Con el fin de orientar a los docentes a realizar un análisis crítico de los mismos. Esta reflexión implica también enfatizar en la importancia que conlleva el dar continuidad a dichos resultados, al establecer estrategias de mejora continua, en beneficio de los aprendizajes de los estudiantes.

El tema de la evaluación es muy complejo ya que contiene una variedad de conceptos, funciones y finalidades. Al realizar una búsqueda sobre estudios vinculados a esta temática a nivel nacional se encontraron los siguientes:

Dicovskiy Riobóo (2019) realizó un trabajo investigativo a nivel de doctorado sobre "Calidad del Rendimiento Académico de los Estudiantes en la carrera de Ingeniería Agroindustrial, de la UNI Región Norte, período 2005-2018". El autor de la investigación en las conclusiones plantea:

Las acciones estratégicas para mejorar el rendimiento académico se deben implementar en tres niveles: los estudiantes, los docentes y las autoridades académicas universitarias. Estas acciones deben estar orientadas a incidir en los tres constructos: motivación, metacognición y género, ya que son los que estuvieron afectando el rendimiento académico, e indirectamente la calidad educativa. (p.201)

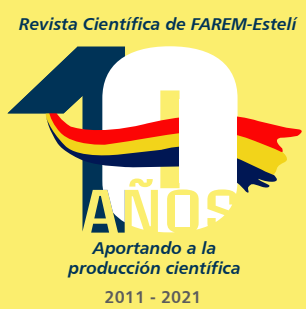


En una investigación presentada, a nivel de doctorado, por Martínez Ramos (2019) y titulada "Modelo de Gestión de Calidad del Rendimiento Académico para la Carrera de Ingeniería en Ciencias de la Computación, de la FAREM Matagalpa, UNAN-Managua, en el periodo 2013 - 2017.", se plantean los siguientes resultados:

El modelo de gestión tiene como objetivo principal mejorar el rendimiento académico de los estudiantes de Ing. en Ciencias de la Computación, en la FAREM Matagalpa, disminuyendo la incidencia negativa de los factores personales, sociales, académicos e institucionales en las calificaciones. Esto se puede lograr solo si estudiantes, docentes y autoridades contribuyen de forma óptima en la implementación de las estrategias propuestas (se enfoca en cuatro componentes principales: infraestructura y equipamiento, recursos humanos, estrategias de gestión y planes de estudio y programas). (pp.124-125)

Se evidencia en las investigaciones antes señaladas, el planteamiento de diversos mecanismos para la evaluación. La importancia de dar seguimiento a las diferentes propuestas contenidas, ya sea en un sistema o modelo de evaluación, como herramientas aplicables y útiles para orientar los procesos de evaluación y mejoramiento de los resultados de la institución (a través de acciones estratégicas o componentes).

Por lo antes mencionado, se considera que este estudio es pertinente. La mejora de la calidad del proceso educativo, estará en dependencia de las decisiones acertadas que realicen los directivos y/o docentes de la institución.

\section{MATERIALES Y MÉTODOS}

La metodología empleada para elaborar este artículo consistió en una revisión bibliográfica de fuentes relacionadas con la evaluación en el ámbito educativo. Para ello se consultaron las Bases de Datos suscritas de la UNANManagua, el Repositorio Centroamericano y Google Académico. Las fuentes consultadas fueron libros, artículos de revistas, tesis doctorales y el modelo educativo de la UNAN-Managua. Los datos encontrados han permitido realizar el estudio y análisis de contenido de la definición de evaluación, sus características, modalidades, paradigmas, así como del informe de rendimiento académico. Este estudio muestra la importancia de la evaluación del rendimiento académico al convertirse en un proceso de reflexión, donde el estudiante tome conciencia de sus metas y el docente sea la guía hacia el logro de sus aprendizajes.

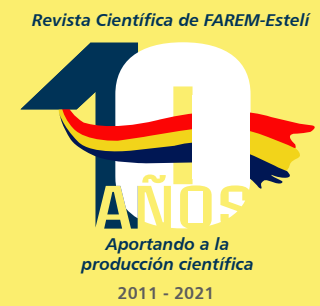




\section{RESULTADOS Y DISCUSIÓN}

Los avances científicos y tecnológicos en el mundo conllevan a diferentes formas de proyección profesional, personal y laboral. En el área del aprendizaje las mejoras se dirigen a la acción formativa de los estudiantes, es así que las instituciones educativas tienen un gran reto para responder a las exigencias y demandas educativas en un contexto lleno de grandes desafíos, como es la formación de profesionales que estén al servicio de la sociedad.

En relación a la praxis universitaria que se implementa en los salones de clase, una parte corresponde a validación de lo aprendido por los estudiantes en formación, la cual se lleva a cabo como evidencia, para la continuidad o certificación de los educandos. (Rueda Beltrán, 2014).

Para iniciar con una aproximación del tema de estudio, es necesario identificar el concepto de evaluación. En el ámbito educativo se encuentran diferentes definiciones, es así, que adquiere diversas connotaciones, tal como lo expresan los siguientes enunciados:

Según García Ramos (1989, citado en Castillo Arredondo y Caberizo Diago, 2010), la evaluación es: "un proceso sistemático de identificación, recogida y tratamiento de datos sobre elementos y hechos educativos con el objetivo de valorarlos primero, y sobre dicha valoración tomar decisiones" (p.7).

Otra definición de evaluación es: "el proceso sistemático de recogida y análisis de información destinado a describir la realidad y emitir juicios de valor sobre su adecuación a un patrón o criterio de referencia explícito o implícito, como base previa para la toma de decisiones" (Gairín Sallán, 2009, p.21).

Se destaca, en cada planteamiento de los autores, a la evaluación como un proceso, que se lleva a cabo para la toma de decisiones, con el fin de aportar a la mejora educativa.

Para evitar la confusión del uso de los términos 'datos' y de 'información', se hace la siguiente aclaración, los datos se recolectan, la información se construye en función del objetivo, a partir de los datos recolectados (análisis).

\section{Diferencia entre medir y evaluar}

La evaluación en la educación es un componente esencial de la valoración del esfuerzo que hace el sistema educativo para la formación de sus estudiantes con calidad. 
El vocablo de evaluación, en diversas ocasiones, se ha asumido como sinónimo de medición. Sin embargo, son términos distintos, a continuación, se presentan las definiciones para cada una de ellas:

La medición consiste en la asignación de números que permiten expresar en términos cuantitativos el grado en que un estudiante posee una determinada característica (...). Por evaluación se entiende la emisión de juicios de valor una vez que se ha recopilado suficiente información acerca del estudiante. (...). Por lo tanto, se puede afirmar que la medición es un componente importante de la evaluación, pero no se puede reducir la evaluación a una simple medición. (Coordinación Educativa y Cultural Centroamericana (CECC), 2008, p.59)

De manera que la medición es una actividad más específica que evaluar, adjudicar un valor numérico. La evaluación de los aprendizajes de los estudiantes es una práctica que con frecuencia se limita al proceso de medición con fines de acreditación del semestre académico o promoción del año lectivo, sin hacer énfasis en las razones por las cuales fallaron los estudiantes y como ayudar a superar estas limitantes.

La medición implica recolectar datos y cuantificar. La evaluación implica también el análisis de los datos para llegar a una valoración crítica de dichos datos en un contexto determinado (el objetivo del análisis).

\section{Características de la evaluación}

Desde el punto de vista de Santos Guerra (2009, pp.67-68) las principales características de la evaluación son:

1. Tiene en cuenta el contexto. (...)

2. Está atenta al proceso y no solo a los resultados. Los procesos y resultados no son excluyentes ni necesariamente contradictorios. (...)

3. Da voz a los participantes en condiciones de libertad. (...)

4. Usa métodos diversos y suficientemente sensibles para captar la complejidad de los fenómenos que se producen en la escuela. (...)

5. Está atenta a los valores, no circunscribiendo su preocupación a la búsqueda de indicadores de carácter técnico. Y está abierta a valores, no sólo en el contenido de la evaluación sino en el mismo proceso evaluador.

6. No convierte la complejidad en simplificaciones abusivas, a través de números y estadísticas.

7. Se realiza a partir de una negociación en la que esté garantizada la defensa de los intereses de toda la comunidad y no los de grupos determinados. (...)

8. Tiene un carácter estructural, ya que se preocupa por el Centro como unidad funcional. (...)

9. Se expresa, a través de los informes, en un lenguaje accesible a los destinatarios. (...)

10. Es educativa al menos, en dos sentidos: el primero, porque se ocupa del valor educativo de la acción escolar. El segundo, porque ella misma

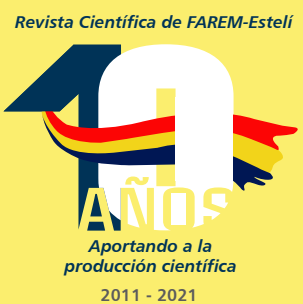


pretende ser educativa al impulsar el diálogo, la participación y la reflexión compartida sobre la mejora.

11.Pretende ser una plataforma de participación y diálogo sobre la enseñanza y la educación.

12.Está comprometida con la sociedad, no solo con el Centro evaluado. (...)

En las características antes citadas se realiza un análisis de lo que implica la evaluación en el proceso educativo, en el cual se toma en cuenta la opinión de los participantes (docentes, estudiantes, directivos escolares, entre otros) y se refleja en un informe, con el fin de mejorar la formación de los estudiantes en la institución.

\section{Modalidades de la evaluación}

Las clasificaciones de las modalidades de evaluación son variadas, por los criterios que pueden ser utilizados, en la siguiente tabla se muestra un resumen:

Tabla 1: Modalidades de evaluación Proceso dinámico, abierto y contextualizado

\begin{tabular}{|c|c|c|c|}
\hline Modalidad & Inicial & Procesual & Final \\
\hline Momento & Antes de... & Durante... & Después de... \\
\hline Funciones & Diagnóstica & Formativa & Sumativa \\
\hline Aplicaciones & \multicolumn{3}{|c|}{$\begin{array}{l}\text { - Evaluación de los estudiantes } \\
\text { - Evaluación de los docentes/de los centros } \\
\text { - Evaluación de los procesos } \\
\text { - Evaluación del sistema educativo }\end{array}$} \\
\hline Ejecutores & \multicolumn{3}{|c|}{$\begin{array}{l}\text { - Docentes } \\
\text { - Estudiantes } \\
\text { - Administración Educativa }\end{array}$} \\
\hline
\end{tabular}

Fuente: Castillo Arredondo y Cabrerizo Diago (2010, p.33)

Según la finalidad o funciones atribuidas a la evaluación, estas varían en función de las necesidades del proceso educativo, al respecto Castillo Arredondo y Cabrerizo Diago (2010, pp.36-39) señalan las siguientes:

1. Evaluación diagnóstica: Su finalidad es que el docente inicie el proceso educativo con un conocimiento real de las características de sus estudiantes, tanto en lo personal como en lo académico. (...).

2. Evaluación formativa: Es la evaluación que sirve como estrategia de mejora para ajustar y regular sobre la marcha los procesos educativos, de cara a conseguir los objetivos previstos y las competencias básicas establecidas. (...).

3. Evaluación sumativa: Se aplica esta evaluación al final de un periodo de tiempo determinado como comprobación de los logros alcanzados en

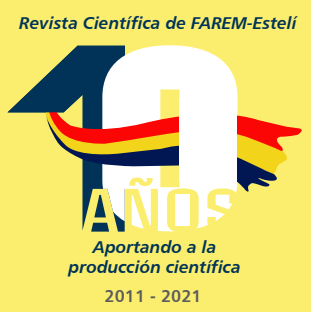


ese periodo. Se pretende determinar la valía final del mismo, el grado de aprovechamiento del estudiante y el grado de consecución de los objetivos propuestos y de las competencias básicas establecidas. La evaluación final tiene una función sancionadora, en la medida en que permite decidir el aprobado o no aprobado de una asignatura; la promoción o no al siguiente curso; o la obtención o no de una determinada titulación.

En la evaluación sumativa cobra presencia y significado la intencionalidad de las evaluaciones anteriores: inicial-diagnóstica y procesual-formativa, y está muy influenciada por ellas. Por otra parte, los resultados de la evaluación sumativa pueden y deben ser el punto de arranque de la evaluación diagnóstica del siguiente periodo escolar.

En la actividad educativa se llevan a cabo diferentes evaluaciones de inicio a fin, es así que se divide en tres categorías:

La evaluación diagnóstica permite caracterizar al grupo de estudiantes, de manera que el docente planifique su asignatura atendiendo a las necesidades de formación de los educandos y reflexione sobre la forma de llevar a cabo la docencia.

La evaluación formativa es fundamental para mejorar la calidad educativa. El docente debe promover evaluaciones en donde la participación activa de los educandos es primordial para el logro de aprendizajes.

En la evaluación para el aprendizaje, los docentes reúnen evidencias de lo que los estudiantes saben, saben cómo, muestran cómo y hacen (fortalezas), así como aquellos aspectos que representan áreas de oportunidad para mejorar su aprendizaje, por lo que los docentes son facilitadores, que junto con los estudiantes, buscan e interpretan la evidencia con la intención de usarla y determinar en dónde están los estudiantes en su aprendizaje, a dónde deben ir y la mejor manera de ayudarlos a llegar ahí. (Coordinación de Desarrollo Educativo e Innovación Curricular, UNAM, 2020, p.42)

Este tipo de evaluación permitirá diseñar un camino de oportunidades para el aprendizaje de los estudiantes, realizar una selección adecuada de las estrategias a implementar en la clase con énfasis en el éxito escolar.

La UNAN-Managua, en su Modelo Educativo, Normativa y Metodología para la Planificación Curricular (2011, pp.32-33) plantea los siguientes aspectos sobre la evaluación:

La evaluación se concibe como parte del proceso enseñanza-aprendizaje, y, por tanto, su objetivo primordial no es la evaluación sumativa de los conocimientos adquiridos por los estudiantes.

La evaluación se entiende como la reunión de información de forma ordenada y sistemática que permite la toma de decisiones que posibilitan reconducir, 
redefinir o bien reorientar el desarrollo del proceso educativo. La auto-reflexión y reflexión sobre las causas de las deficiencias encontradas facilitan la toma de decisiones que posibilitan reconducir el proceso, y por tanto desarrollar una evaluación formativa. Esto permite que los estudiantes conozcan cómo han avanzado y en qué lugar del proceso se encuentran, conocer sus dificultades y qué pueden hacer para mejorar. Esto implica la creación de espacios, ambientes e instrumentos que favorezcan la heteroevaluación, autoevaluación y coevaluación.

La evaluación sumativa es la que se hace como resultado de la actividad académica desarrollada, para determinar los logros alcanzados por los estudiantes y certificar su continuidad en su formación académica. Es necesario que los insumos suministrados en esta evaluación, sean retomados en el siguiente periodo escolar.

"La evaluación educativa depende de la metodología utilizada, la calidad del proceso y el uso que se hace de los resultados" (Coordinación de Desarrollo Educativo e Innovación Curricular, UNAM, 2020, p.22).

De acuerdo con el planteamiento de Sánchez Alvarado y Orozco Alvarado (2019):

Un camino para abordar la evaluación auténtica es convertir la tarea de evaluar en una actividad cualitativa y formativa que permita devolver la mirada hacia sus protagonistas, hacia los procesos y las acciones para el desarrollo de competencias y potencialidades que han de tener lugar en las aulas, para tomar consciencia sobre el curso de los procesos, valorarlos y proporcionar ayuda a los estudiantes en el momento requerido. (p.6)

La evaluación procesual en su función formativa consiste en la valoración, a través de la recogida continua y sistemática de datos, del funcionamiento de un centro, de un programa educativo, del proceso educativo de un estudiante, etc., a lo largo de un periodo de tiempo prefijado para la consecución de las metas u objetivos propuestos. La evaluación procesual sirve como estrategia de mejora para ajustar y regular sobre la marcha los procesos educativos (de ahí su nombre). (Castillo Arredondo y Cabrerizo Diago, 2010, p.35)

\section{Paradigmas de la evaluación}

Existen dos grandes paradigmas que se conocen con el nombre de positivista (cuantitativo) y naturalista (cualitativo), como plantea la Coordinación Educativa y Cultural Centroamericana (2008, pp.66-67), a continuación:

Las evaluaciones realizadas por protagonistas del primer grupo, conocidas como evaluaciones tradicionales, se caracterizan por hacer énfasis en la evaluación de los productos del aprendizaje. (...).

Se evalúan básicamente los conocimientos y se califica numéricamente. Se evalúa para seleccionar a los estudiantes en forma unidireccional, o sea, es 
solo el docente quien lo hace. Se privilegian las mediciones de los logros, se enfatiza en la evaluación del estudiante, se evalúan principalmente los efectos observables. (...).

Las evaluaciones del segundo grupo, o sea, las ubicadas en la corriente alternativa, se distinguen porque reflejan un creciente interés por tomar en cuenta el proceso de enseñanza y aprendizaje, así como el producto. Este enfoque se le conoce también como enfoque innovador o actual.

Se considera como una alternativa en el campo de la evaluación, porque la tarea actual de los docentes consiste en producir iniciativas, con el supuesto de variar los sistemas de evaluación para que potencien el desarrollo integral del estudiante, con la intención de incidir en forma favorable en el aprendizaje significativo para que su aplicación sea posible en el contexto de las aulas.

Las posiciones teóricas de los dos paradigmas son distintas. Sin embargo, se evidencia el aporte que brindan a la evaluación. Ambos paradigmas se complementan entre sí, contribuyen a realizar una evaluación más objetiva de los aprendizajes de los estudiantes, durante la acción educativa.

El análisis de la evaluación realizada por los docentes a los estudiantes constituye una tarea fundamental de los sistemas educativos para la promoción de la mejora de la calidad. Se pueden determinar las fortalezas y debilidades en el proceso educativo, justo a partir del análisis crítico de sus resultados (rendimiento académico).

Todo esfuerzo de evaluación debe estar diseñado con propósitos claros, definido el uso que se le dará a los resultados de la valoración en beneficio del aprendizaje de los estudiantes, aportando a la calidad educativa de la institución.

\section{Rendimiento académico}

La evaluación forma parte de los factores para garantizar la calidad de los procesos educativos y al mismo tiempo, un medio idóneo que facilita la mejora permanente de los mismos.

Se considera ineludible la elaboración del informe de rendimiento académico, retomar esos resultados, que son fundamentales para conocer la efectividad del trabajo académico desarrollado en la institución y constatar los efectos producidos en el proceso de aprendizaje de los estudiantes.

Como definición de rendimiento académico, según Puche (1999, citado en Sánchez de Gallardo y Pirela de Faría, 2006) "es un proceso multidisciplinario donde intervienen la cuantificación y la cualificación del aprendizaje en el desarrollo cognitivo, afectivo y actitudinal que demuestra el estudiante en la resolución de problemas asociados al logro de los objetivos programáticos propuestos" (p.13).

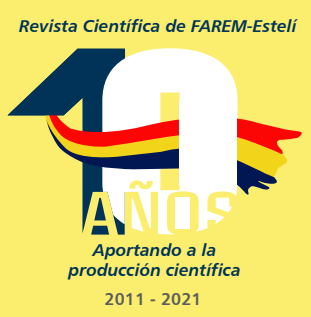


El informe de rendimiento académico debe constituirse en una herramienta de realimentación para el análisis minucioso de los aspectos de la evaluación final que resultaron satisfactorios y es necesario fortalecer y aquellos que serán precisos modificar.

En función del análisis del rendimiento académico se pueden elaborar propuestas de mejora para la planificación del siguiente semestre académico y/o año lectivo, en beneficio del aprendizaje de los estudiantes. De igual manera dichas propuestas puedan estar extendidas a todos los años de la carrea y no a una asignatura en particular.

Lo cual permitirá garantizar la coherencia entre las mediaciones que se desarrollen en los grupos de un mismo nivel educativo. Esto podrá llevar a fortalecer las debilidades encontradas, mejorar la práctica docente y por ende la calidad educativa por la que trabaja toda institución de educación.

En Venezuela, Guzmán Zamora (2020), escribió un artículo denominado "Motivación escolar: metas académicas, estilos atribucionales y rendimiento académico en estudiantes de educación media" en donde manifiesta que:

El estudio del rendimiento académico es un campo de investigación relevante a nivel educativo, pues identificar los elementos que pueden mejorar o dificultar el rendimiento académico facilita el diseño de estrategias de intervención y de políticas educativas. En tal sentido, un importante número de investigaciones se ha enfocado en el estudio de aquellas variables que representan un papel importante en el desempeño académico, vislumbrando la incidencia que tienen los factores motivacionales sobre éste. (p.291)

Por lo tanto, es primordial valorar el grado de cumplimiento que realicen los docentes con la elaboración del informe de rendimiento académico de manera subjetiva y el seguimiento que se le dé a los resultados encontrados.

\section{Aspectos a considerar en la evaluación}

Se considera que las evaluaciones para el aprendizaje son de calidad cuando los docentes pueden emplearlas para la toma de decisiones sobre el aprendizaje de los estudiantes, con suficiente grado de precisión y especificidad, para brindar realimentación a los estudiantes y que tenga el poder de brindarle información para planear la próxima etapa de aprendizaje. (Coordinación de Desarrollo Educativo e Innovación Curricular, UNAM, 2020, p.46)

Es importante destacar en la evaluación los aportes que puede brindar al recolectar datos sobre cada una de las partes que integran el proceso educativo. Los datos, al ser interpretados, nos permitirán elaborar la información útil para mejorar la calidad del aprendizaje de los estudiantes.

Los docentes, al realizar las evaluaciones de los estudiantes, deben plantearse una serie de preguntas, referidas a ¿̇cómo estudiar esos resultados?, ¿̇qué

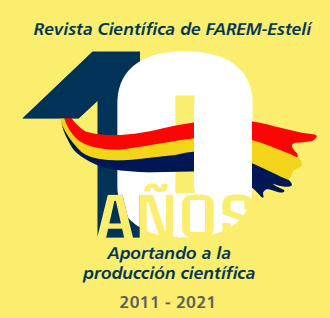


utilidad tendrán los resultados?, ¿̇cómo contribuirán las evaluaciones en el fortalecimiento del aprendizaje de los estudiantes?, ¿̇qué reflexiones surgieron de la práctica docente, con el fin de realizar los ajustes necesarios atendiendo las necesidades de los estudiantes? Es fundamental comunicar a los estudiantes, desde el inicio del curso académico, los objetivos de aprendizaje que se espera alcancen.

\section{CONCLUSIONES}

En este trabajo se ha procurado enfatizar el valor de la evaluación, al ser un instrumento para cambiar y mejorar el proceso educativo en el aula de clase. Cuando la evaluación es realizada de manera crítica y reflexiva incide notablemente en la calidad del proceso de aprendizaje y por ende en la formación de los estudiantes.

Como experiencia en la universidad (UNAN-Managua) y particularmente en nuestra Facultad Regional Multidisciplinaria, Estelí (FAREM-Estelí), en cada asignatura, se elabora el informe de rendimiento académico como parte de la evaluación final del proceso académico. Dicho informe debe ser diseñado desde la visión de la mejora continua al establecer estrategias, en beneficio del aprendizaje de los estudiantes, y no únicamente como una valoración de los resultados obtenidos en el que se describan las debilidades encontradas. La evaluación busca mejorar el acto educativo en las instituciones y el aprendizaje de los estudiantes, al permitir a los docentes obtener las evidencias para guiar a los educandos por medio de la identificación de las fortalezas y debilidades.

Se ha enfocado en la evaluación formativa y sumativa en este estudio, en el entendimiento que la evaluación formativa es procesual, para mejorar el aprendizaje de los estudiantes al iniciar un semestre académico o año lectivo. La evaluación con fines de mejora debe ser un proceso constante en las instituciones educativas.

\section{REFERENCIAS BIBLIOGRÁFICAS}

Castillo Arredondo, S., \& Cabrerizo Diago, J. (2010). Evaluación educativa de aprendizajes y competencias. Madrid, España: PEARSON EDUCACIÓN. S.A.

Coordinación de Desarrollo Educativo e Innovación Curricular, UNAM. (2020). Evaluación del y para el aprendizaje: instrumentos y estrategias

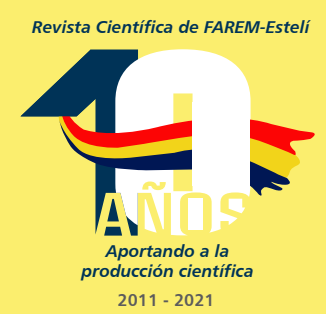


(Primera ed.). Ciudad de México, México. Obtenido de https://www. codeic.unam.mx/wp-content/uploads/2020/01/Evaluacion_del_y_para_ el_aprendizaje.pdf

Coordinación Educativa y Cultural Centroamericana (CECC). (2008). Evaluación Educativa. Cuatro enfoques (Primera ed.). San José, Costa Rica.

De Miguel, N. Z. (2012). La evaluación como proceso sistemático para la mejora educativa. España: Ministerio de Educación de España. Obtenido de https://elibro.net/es/ereader/unanmanagua/49323? page $=16$

Dicovskiy Riobóo, L. M. (2019). Tesis Doctoral. Calidad del Rendimiento Académico de los Estudiantes en la carrera de Ingeniería Agroindustrial, de la UNI Región Norte, período 2005-2018, 271. Estelí, Nicaragua. Obtenido de https://repositorio.unan.edu.ni/12311/1/5885.pdf

Gairín Sallán, J. (2009). Usos y abusos en la evaluación. En M. d. Profesional, Nuevas Funciones de la Evaluación (pág. 268). España.

Guzmán Zamora, N. (2020). Motivación escolar: metas académicas, estilos atribucionales y rendimiento académico en estudiantes de educación media. Archivos Venezolanos de Farmacología y Terapéutica, 39(3), 290-295. Obtenido de http://web.b.ebscohost.com/ehost/ pdfviewer/pdfviewer? vid =4\&sid =f6 $18 f f 16-204 d-4$ cf8-bb55b2e49bb82a7a\%40pdc-v-sessmgr03

Martínez Ramos, G. R. (2019). Tesis Doctoral. Modelo de Gestión de Calidad del Rendimiento Académico para la Carrera de Ingeniería en Ciencias de la Computación, de la FAREM Matagalpa, UNAN-Managua, en el periodo 2013 - 2017, 253. Estelí, Nicaragua. Obtenido de https://repositorio. unan.edu.ni/12310/1/5883.pdf

Rueda Beltrán, M. (2014). La evaluación educativa: análisis de sus prácticas. Ediciones Díaz de Santos. Obtenido de https://elibro.net/es/ereader/ unanmanagua $/ 62805$ ? page $=327$

Sánchez Alvarado, A. F., \& Orozco Alvarado, J. C. (2019). Las competencias y la evaluación. Hacia un modelo de evaluación auténtica de los aprendizajes. Revista Científica de FAREM-Estelí. Medio ambiente, tecnología y desarrollo humano(32), 12. Obtenido de https://rcientificaesteli.unan.edu.ni/index. $\mathrm{php} / \mathrm{RCientifica/article/view/960/938}$

Sánchez de Gallardo, M., \& Pirela de Faría, L. (2006). Motivaciones sociales y rendimiento académico en estudiantes de educación. Revista de Ciencias Sociales. Universidad del Zulia, 12(1), 22. Obtenido de https://elibro.net/ es/ereader/unanmanagua/ 17208 ? page $=13$

Santos Guerra, M. Á. (2009). La Evaluación Institucional como autorregulación. En M. d. Profesional, Nuevas Funciones de la Evaluación (pág. 268). España.

Universidad Nacional Autónoma de Nicaragua,Managua. (2011). Modelo Educativo, Normativa y Metodología para la Planificación Curricular. 134. Nicaragua.

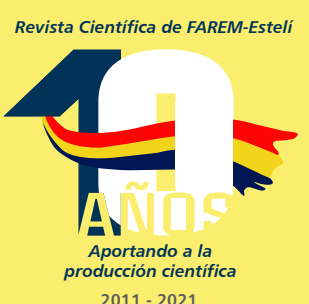

